\title{
Cell Curvature and Far-Field Superconvergence in Numerical Solutions of Electromagnetic Integral Equations
}

\author{
Andrew F. Peterson \\ School of ECE, Georgia Institute of Technology, Atlanta, GA 30332, USA \\ Correspondence should be addressed to Andrew F. Peterson; peterson@ece.gatech.edu
}

Received 5 January 2016; Accepted 3 April 2016

Academic Editor: Toni Björninen

Copyright ( 2016 Andrew F. Peterson. This is an open access article distributed under the Creative Commons Attribution License, which permits unrestricted use, distribution, and reproduction in any medium, provided the original work is properly cited.

\begin{abstract}
Two curved targets are used to explore far-field superconvergence effects arising in numerical solutions of the electric-field and magnetic-field integral equations. Three different orders of basis and testing functions are used to discretize these equations, and three different types of target models (flat facets, quadratic-curved facets, and cubic-curved facets) are employed. Ideal far-field convergence rates are only observed when the model curvature is one degree higher than the basis order.
\end{abstract}

\section{Introduction}

Electromagnetic analysis has often exploited "variational” or "stationary" techniques in an attempt to obtain results that exhibit smaller error or faster convergence than might otherwise be obtained for a given order of approximation. Representations in terms of degree $p$ polynomials are expected to produce error rates of $O\left(h^{p+1}\right)$ as $h \rightarrow 0$, where $h$ is the characteristic cell dimension. For instance, a linear representation should produce $O\left(h^{2}\right)$ convergence. Superconvergence occurs when the error decreases at a faster rate. Galerkin method-of-moments (MoM) procedures, which involve the use of identical functions for expanding the unknown surface currents and enforcing the integral equation, sometimes exhibit superconvergence in the far-zone fields or scattering cross section (SCS) [1-4]. Theoretical SCS convergence rates were established by Warnick for the electric-field integral equation (EFIE) and magnetic-field integral equation (MFIE) [5] and are reported below. One example of superconvergence arises with the popular Rao-Wilton-Glisson (RWG) solutions of the EFIE [6], which usually yield SCS error rates of $O\left(h^{2}\right)$ $O\left(h^{3}\right)$ in practice instead of the $O(h)$ rate expected from basis functions that are only complete to degree 0 .

When and where superconvergence occurs are not always understood. It was demonstrated in [7] that mixed-order divergence-conforming basis functions often produce superconvergent far fields with the EFIE, but polynomial-complete basis functions apparently do not. In a complementary manner, polynomial-complete basis functions seem to produce superconvergent far fields with the MFIE, while mixed-order bases do not. Thus, the MFIE requires types of basis functions different than the EFIE for optimal SCS errors. Furthermore, ideal convergence rates should not be expected if target models are not accurate enough, for instance, if flat facets are used to model curved surfaces. Convergence rates are usually slower if the problem geometry contains edges or corners where current or charge densities may be singular, unless special basis functions containing the proper behavior are employed [8]. The purpose of the following is to attempt to clarify conditions under which one can reasonably expect to achieve the ideal convergence rates of [5]. Primarily, we investigate the issue of curved-cell models and the required accuracy in the testing integrals associated with the MoM procedure for perfectly conducting targets that are spheres or toroids.

We use mixed-order divergence-conforming vector basis functions of the Nedelec variety [9] on curved triangular patches to discretize the EFIE. The RWG basis functions [6] are the lowest-order $(p=0.5)$ members of this family. Hierarchical versions of these functions are described in [10] 
and were implemented in a Galerkin scheme. Functions of orders $p=0.5, p=1.5$, and $p=2.5$ are considered and are reported as follows.

Local Definition of the Hierarchical Divergence-Conforming Vector Basis Functions Used with the EFIE, in Simplex Coordinates $(u, v, w)$ on a Standard Triangle. The Edge-Based Functions Straddle Adjacent Cells

Edge-based base vectors $(p=0.5)$ are

$$
\begin{aligned}
& \bar{\Lambda}_{1}=(u-1) \widehat{u}+v \widehat{v}, \\
& \bar{\Lambda}_{2}=u \widehat{u}+(v-1) \widehat{v}, \\
& \bar{\Lambda}_{3}=u \widehat{u}+v \widehat{v} .
\end{aligned}
$$

Order $p=1.5$ includes three additional edge-based functions

$$
\begin{aligned}
& \sqrt{3}(v-w) \bar{\Lambda}_{1}, \\
& \sqrt{3}(w-u) \bar{\Lambda}_{2}, \\
& \sqrt{3}(u-v) \bar{\Lambda}_{3}
\end{aligned}
$$

and 2 cell-based functions

$$
\begin{aligned}
& 2 \sqrt{3} u \bar{\Lambda}_{1}, \\
& 2 \sqrt{3} v \bar{\Lambda}_{2} .
\end{aligned}
$$

Order $p=2.5$ includes three additional edge-based functions

$$
\begin{aligned}
& \sqrt{5}\left\{\left(3(v-w)^{2}-1\right) / 2-(u / 2)(u-2)\right\} \bar{\Lambda}_{1}, \\
& \sqrt{5}\left\{\left(3(w-u)^{2}-1\right) / 2-(v / 2)(v-2)\right\} \bar{\Lambda}_{2}, \\
& \sqrt{5}\left\{\left(3(u-v)^{2}-1\right) / 2-(w / 2)(w-2)\right\} \bar{\Lambda}_{3}
\end{aligned}
$$

and 4 cell-based functions

$$
\begin{aligned}
& 6 \sqrt{5}(v-w) u \bar{\Lambda}_{1}, \\
& 6 \sqrt{5}(w-u) v \bar{\Lambda}_{2}, \\
& 2 \sqrt{3} u(5 u-3) \bar{\Lambda}_{1}, \\
& 2 \sqrt{3} v(5 v-3) \bar{\Lambda}_{2} .
\end{aligned}
$$

Inner integrals were evaluated using adaptive quadrature routines in conjunction with singularity cancellation procedures to return results accurate to some number of decimal places (6 digits for the $p=2.5$ results). Testing integrals were evaluated with a 7-point Gauss rule (that can exactly integrate degree 5 functions) for $p=0.5$ and $p=1.5$ and a 12 -point Gauss rule that can exactly integrate degree 7 polynomials for $p=2.5$. These rules were selected after numerical experimentation showed that higher-order rules do not improve the accuracy or convergence rates of the results. From Warnick's analysis, the error in the SCS obtained from the EFIE under optimal conditions should converge at a rate of $O\left(h^{2 p+2}\right)$, where half-integer indices are used for $p$ to denote the mixed-order functions. For instance, the SCS error associated with $p=0.5$ functions is ideally expected to converge as $O\left(h^{3}\right)$, while that of the $p=1.5$ functions is ideally expected to converge as $O\left(h^{5}\right)$. These are superconvergent rates since they are different from the rates associated with the underlying current density, namely, $O\left(h^{p+0.5}\right)$. It is noteworthy that $O\left(h^{p+0.5}\right)$ rates are observed for SCS values when point-testing is used with the EFIE instead of Galerkin testing [11].

On the other hand, $[7,12,13]$ suggest that the MFIE SCS is not superconvergent unless polynomial-complete bases are employed. So for the MFIE we use polynomial-complete bases of orders $p=0.0$ (constant), $p=1.0$ (linear), and $p=2.0$ (quadratic) on curved triangular patches to treat the MFIE. The $p=1.0$ and $p=2.0$ functions are of the curl-conforming variety and belong to Nedelec's polynomialcomplete spaces [14]. The specific basis functions used are interpolatory and are reported as follows.

Local Definition of the Interpolatory Curl-Conforming Vector Basis Functions Used with the MFIE, in Simplex Coordinates $(u, v, w)$ on a Standard Triangle. The Edge-Based Functions Straddle Adjacent Cells

Order $p=0.0$ includes two constant cell-based functions:

$$
\begin{aligned}
& \bar{\Omega}_{1}=\widehat{u} \\
& \bar{\Omega}_{2}=\widehat{v} .
\end{aligned}
$$

Order $p=1.0$ includes six linear edge-based functions:

$$
\begin{aligned}
& v \widehat{u}, \\
& u \widehat{v}, \\
& u \widehat{w}, \\
& w \widehat{u}, \\
& w \widehat{v}, \\
& v \widehat{w} .
\end{aligned}
$$

Order $p=2.0$ includes twelve quadratic functions, 9 edge-based

$$
\begin{aligned}
& v(1-2 v) \widehat{w} \\
& w(1-2 w) \widehat{u}, \\
& u(1-2 u) \widehat{v} \\
& w(1-2 w) \widehat{v} \\
& u(1-2 u) \widehat{w} \\
& v(1-2 v) \widehat{u} \\
& v w(\widehat{w}-\widehat{v}) \\
& w u(\widehat{u}-\widehat{w}) \\
& u v(\widehat{v}-\widehat{u})
\end{aligned}
$$

and 3 cell-based

$v w \widehat{u}$,

$w u \widehat{v}$,

$u v \widehat{w}$. 


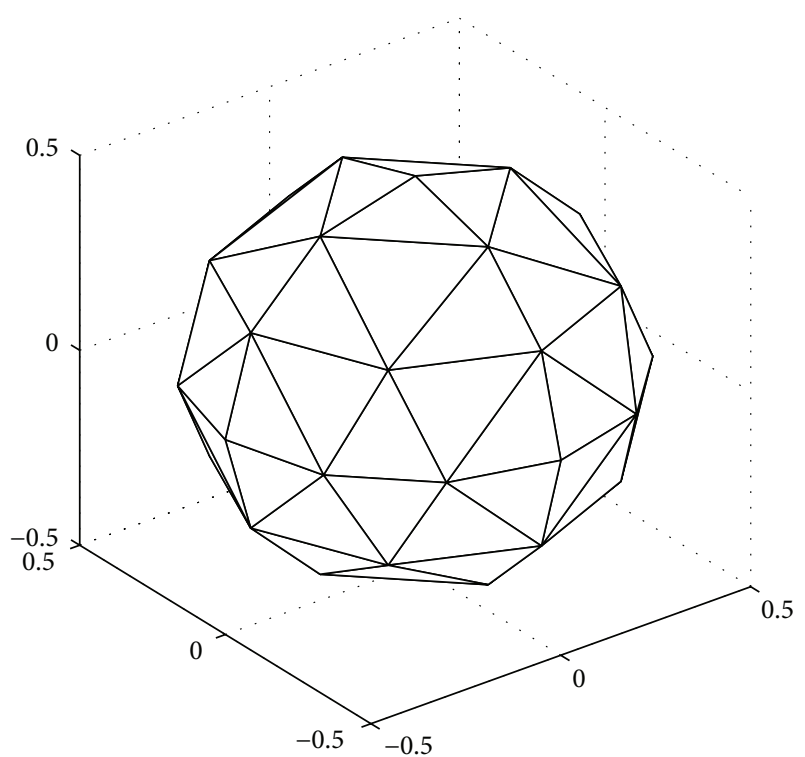

FIGURE 1: Illustration of the triangular-cell sphere models used in the analysis. This model contains 72 flat cells.

As with the EFIE, adaptive quadrature and singularity cancellation are used for the inner integrals. Galerkin testing is implemented with a 7-point Gauss rule, which was demonstrated to be adequate for all three orders. Under ideal conditions, the error in the SCS obtained from the MFIE should converge at a rate of $\mathrm{O}\left(h^{2 p+2}\right)$, where integer indices are used for $p$ [5]. Thus the SCS error associated with $p=0.0$ functions is expected to converge as $O\left(h^{2}\right)$, while that of the $p=1.0$ functions is expected to converge as $O\left(h^{4}\right)$. Note that these rates are one order less than the corresponding rates for the EFIE SCS, based on the extent to which the basis functions are complete to a given degree, but are still superconvergent.

In the following, we investigate the convergence rates that can be obtained for a sphere and toroid target, as a function of the type of curvature used with the model (flat patches, quadratic-curved patches, and cubic-curved patches). This work is intended to build upon and extend the previous work of the author in $[7,11,15]$ in establishing baseline convergence rates for numerical solutions of the EFIE and MFIE.

\section{Results for Scattering from a Sphere}

Triangular-cell models are obtained by dividing a sphere uniformly in $\theta$ between its north pole and its south pole and dividing it uniformly in $\phi$ so that the number of cell edges along the equator is twice the number of divisions in $\theta$. Each octant of the sphere surface is therefore a curved triangle that is divided into smaller triangles of fairly uniform size. Figure 1 shows one example employing 72 flat patches. For curved patches, the cells are mapped from triangles to patches defined by the usual 6 Lagrange points (quadratic) or 10 Lagrange points (cubic), with all the points on the sphere surface. The mapping procedures for the divergence-conforming (EFIE) and curl-conforming (MFIE) bases are described in

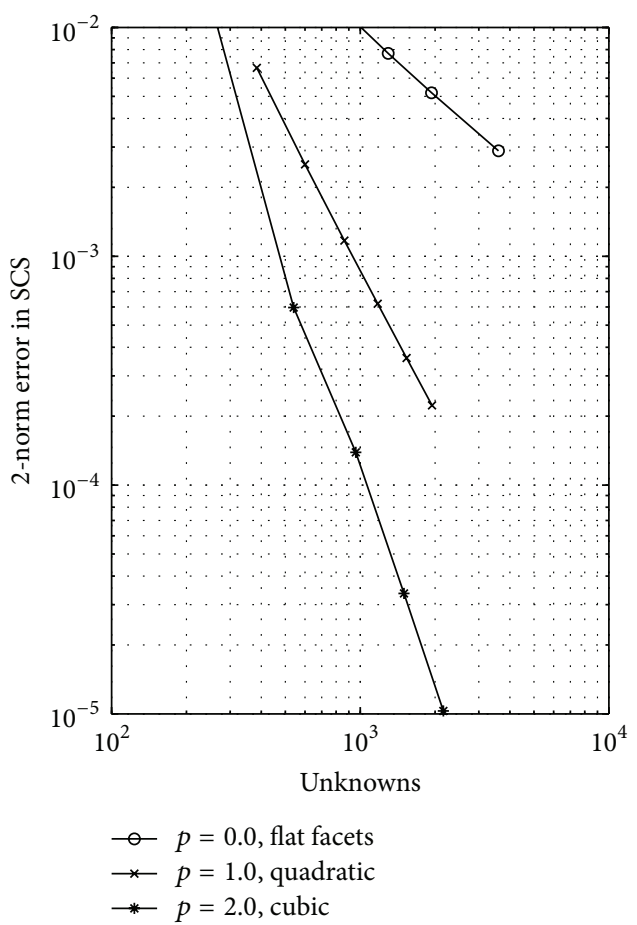

FIGURE 2: Error in the SCS versus number of unknowns for a sphere with $k a=2 \pi$, obtained from the MFIE with Galerkin testing. Results are reported for flat-faceted models used in conjunction with $p=0.0$ basis and test functions, quadratic-curved models used with $p=1.0$ functions, and cubic-curved models used with $p=2.0$ functions.

detail in [16]. The surface area of each subsectional-cell model is normalized to that of the desired sphere.

Relative accuracy is determined by evaluating the error in the bistatic SCS at $5^{\circ}$ increments in $\theta$ and $\phi$ over the entire spherical range, with the 2-norm error computed according to

$$
E=\frac{\sqrt{(1 / N) \sum_{n=1}^{N}\left|e_{n}\right|^{2} \sin \theta_{n}}}{|S C S|_{\max }},
$$

where $e_{n}$ denotes the error at point $n, n=1,2, \ldots, N$, and where $\theta_{n}$ denotes the value of $\theta$ at point $n$. The reference is the exact eigenfunction "Mie series" expansion [1].

Consider a sphere with $k a=2 \pi$ or a surface area of approximately $12.57 \lambda^{2}$. Thus a density of 100 unknowns per square wavelength of surface area is reached when the total number of unknowns equals 1257. Figure 2 shows the error in SCS versus number of unknowns for results obtained from the MFIE. Results are reported for three representations, a flat-faceted model used in conjunction with $p=0.0$ basis and test functions (piecewise constant), a quadratic-curved model used with $p=1.0$ functions (linear), and a cubiccurved model used with $p=2.0$ functions. As expected, the higher-order bases and higher-order target models produce more accurate solutions. Figure 3 shows the same data plotted versus the average edge length used in the models. It is apparent that the $p=0.0$ result closely follows an $O\left(h^{2}\right)$ trajectory, the $p=1.0$ result follows an $O\left(h^{4}\right)$ trajectory, 


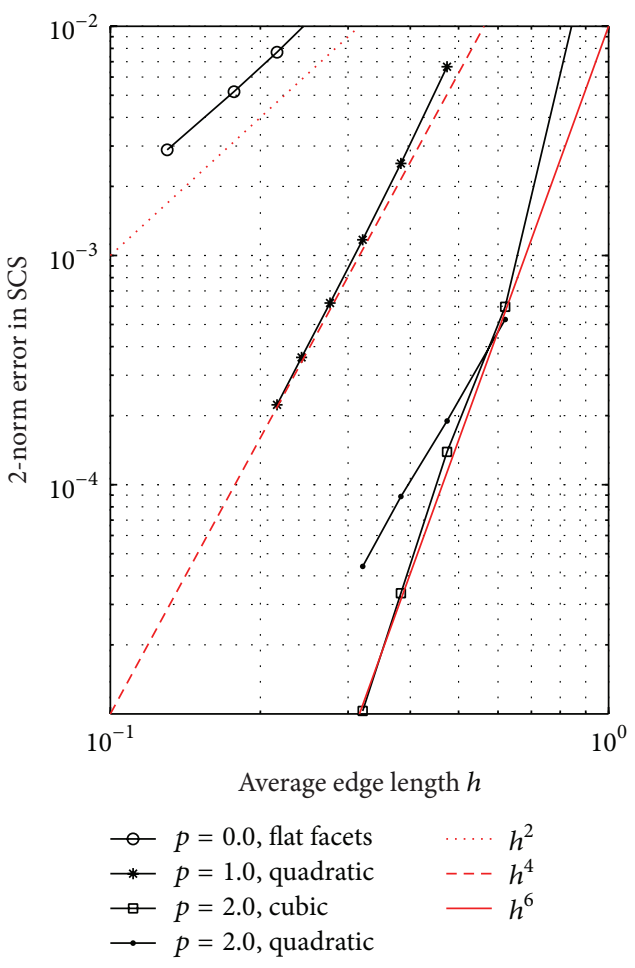

FIGURE 3: Error in the SCS versus the average edge lengths in the models, for a sphere with $k a=2 \pi$. MFIE results from Figure 2 are compared to ideal asymptotic rates of $O\left(h^{2}\right), O\left(h^{4}\right)$, and $O\left(h^{6}\right)$. Also shown is the error obtained with $p=2.0$ basis functions when used with quadratic-curved models.

and the $p=2.0$ result follows an $O\left(h^{6}\right)$ trajectory, for small $h$. These are the ideal rates suggested in [5]. Also shown in Figure 3 is a plot of the corresponding error for the $p=2.0$ case when quadratic-curved patches are used in the model. With the quadratic-patch models, the $p=2.0$ results follow a slower rate, near $O\left(h^{4}\right)$. Similar behavior was observed by the author for other targets (not shown), suggesting the conclusion that $p=2.0$ bases must be used with cubic-patch models to produce optimal convergence rates.

The SCS errors produced using the EFIE are presented in Figures 4 and 5 for the same sphere target. Data were obtained using $p=0.5$ (RWG), $p=1.5$, and $p=2.5$ mixed-order basis and test functions. Figure 4 shows results versus the number of unknowns, with all models represented by cubiccurved patches. In common with the MFIE results, once the number of unknowns is in a reasonable range for a problem of this electrical size, higher-order bases produce more accurate results for a given number of unknowns. Figure 5 shows EFIE results plotted versus the average edge length used in the models, but for the case when the $p=0.5$ results are obtained with flat-faceted models and the $p=1.5$ results are obtained with quadratic-curved models. The $p=0.5$ results follow an $O\left(h^{3}\right)$ trajectory, while the $p=1.5$ results in this case are somewhere between $O\left(h^{4}\right)$ and $O\left(h^{5}\right)$. The $p=2.5$ results appear to follow a trajectory between $O\left(h^{6}\right)$ and $O\left(h^{7}\right)$. Thus, these data are close to the ideal rates of [5], but as observed in $[7,15]$, the ideal rates are not always obtained in practice.

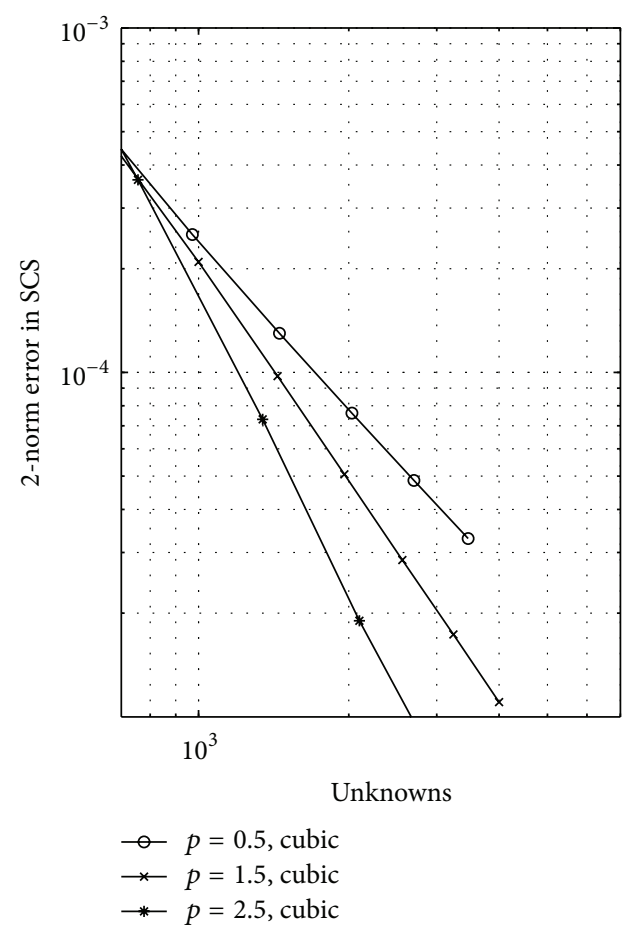

FIGURE 4: Error in the SCS versus number of unknowns for a sphere with $k a=2 \pi$, produced by the EFIE. Results are reported for $p=$ $0.5, p=1.5$, and $p=2.5$ mixed-order divergence-conforming basis and test functions, in conjunction with cubic-curved-patch models.

The author has also investigated the use of $p=2.5$ bases with quadratic-patch sphere models (not shown) but always observed a substantially slower convergence rate for that combination. This suggests that $p=2.5$ bases must be used with cubic-patch models to approach optimal convergence rates.

\section{Results for Scattering from a Torus}

A torus is a simple target that, like a sphere, avoids the complications introduced by edges and corners. For the following comparisons, a torus with major radius of $3 / \pi \lambda$ and minor radius of $1.5 / \pi \lambda$, for a total surface area of $18.0 \lambda^{2}$, is employed. The torus is centered at the origin, parallel to the $x-y$ plane, and is illuminated by a uniform plane wave in the $z$-direction with the electric field polarized along $\hat{x}$. For illustration, Figure 6 shows a torus model containing 196 flat triangular patches.

The torus is divided uniformly along both circumferences into curved quadrilateral cells, which are each divided into two (curved) triangles. The models employed twice as many divisions along the larger circumference as along the smaller and are scaled to the proper surface area. For comparison purposes, a reference solution was obtained using the formulation described in [17], with basis functions complete to polynomial degree 5, and a total of 9216 unknowns. Relative accuracy is determined from (1) by evaluating the error in the bistatic SCS at $5^{\circ}$ increments in $\theta$ and $90^{\circ}$ increments in $\phi$. In the figures for the torus example, a density of 100 unknowns 


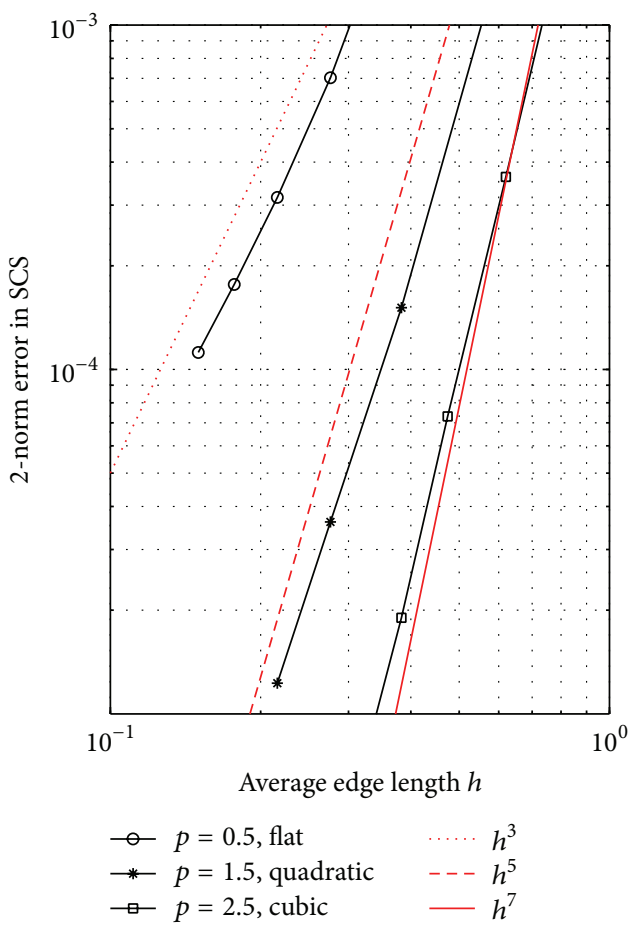

FIGURE 5: Error in the SCS versus the average edge lengths in the models, for a sphere with $k a=2 \pi$. EFIE results are reported for flat-faceted models used in conjunction with $p=0.5$ basis and test functions, quadratic-curved models used with $p=1.5$ functions, and cubic-curved models used with $p=2.5$ functions. Ideal asymptotic rates of $O\left(h^{3}\right), O\left(h^{5}\right)$, and $O\left(h^{7}\right)$ are shown for comparison.

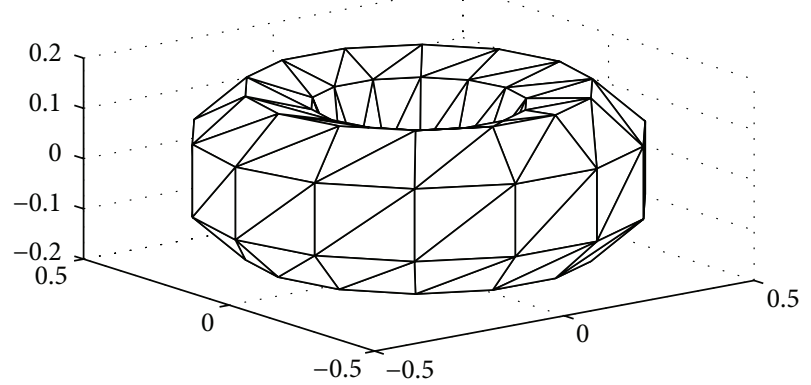

FIGURE 6: Illustration of the triangular-cell torus models used in the analysis. This model contains 196 flat cells.

per square wavelength of surface area is reached when the total number of unknowns equals 1800 .

Figure 7 shows the SCS error produced by the MFIE versus the number of unknowns for flat-faceted models with $p=0.0$ functions, quadratic-curved models with $p=1.0$ functions, and cubic-curved models with $p=2.0$ functions. Figure 8 shows the same data plotted versus the average edge length used in the models. For these results, the $p=0.0$ data exhibit a convergence rate between $O(h)$ and $O\left(h^{2}\right)$, while the $p=1.0$ and $p=2.0$ data closely approximate the ideal trajectories of $O\left(h^{4}\right)$ and $O\left(h^{6}\right)$.

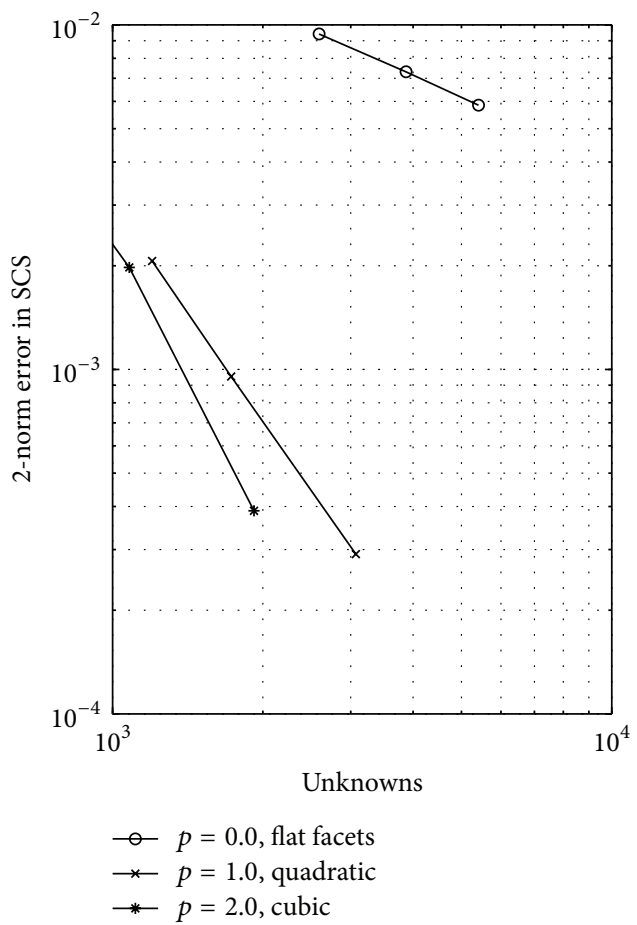

FIGURE 7: Error in the SCS versus number of unknowns for a torus with $k a=3$ and $k b=6$, obtained from the MFIE with Galerkin testing. Results are reported for flat-faceted models used in conjunction with $p=0.0$ basis and test functions, quadratic-curved models used with $p=1.0$ functions, and cubic-curved models used with $p=2.0$ functions.

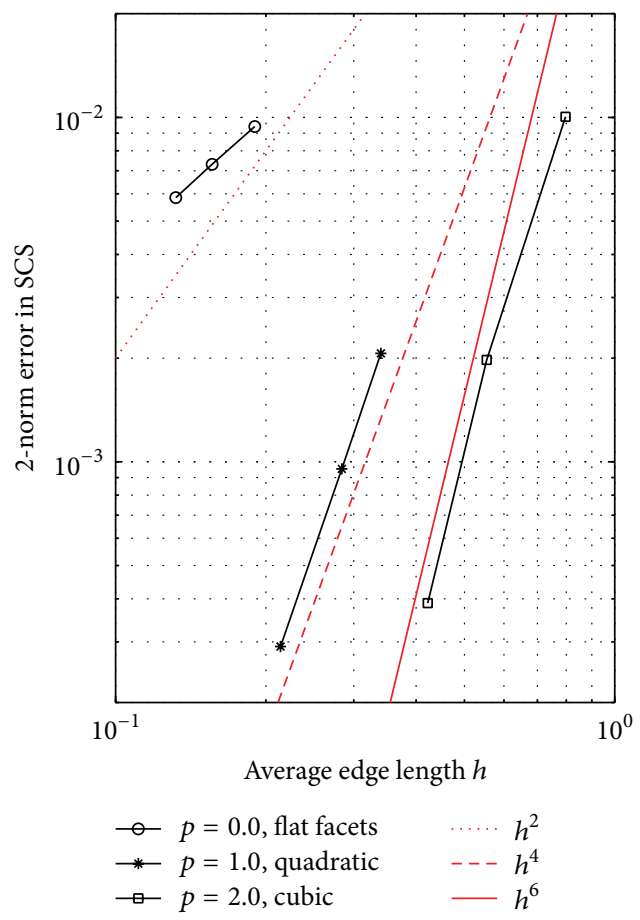

FIGURE 8: Error in the SCS versus the average edge lengths in the models, for the same torus used in Figure 7. MFIE results from Figure 7 are compared to ideal asymptotic rates of $O\left(h^{2}\right), O\left(h^{4}\right)$, and $O\left(h^{6}\right)$. 


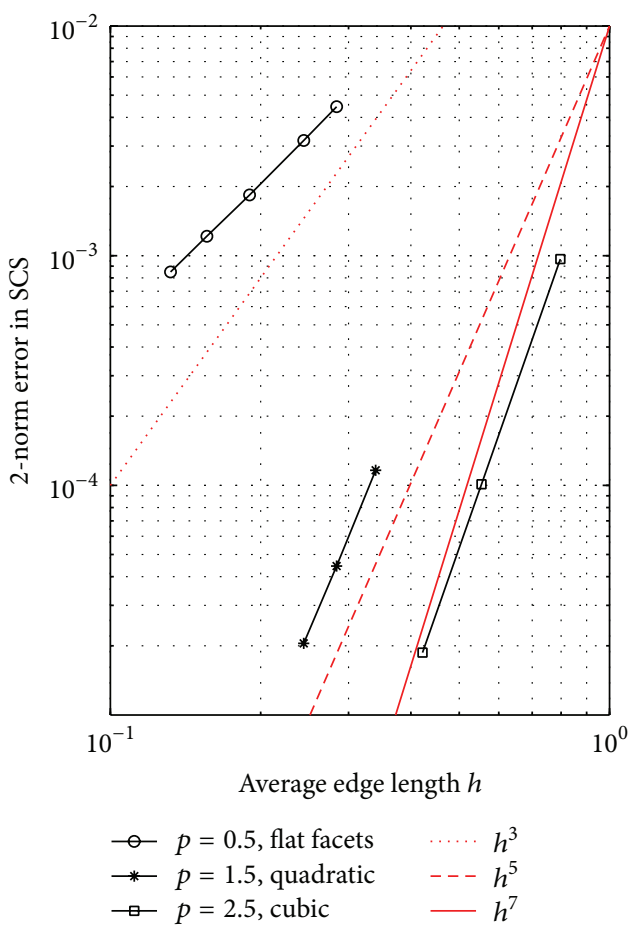

FIGURE 9: Error in the SCS versus the average edge lengths in the models, for the same torus used in Figures 7 and 8. EFIE results are reported for a flat-faceted model used in conjunction with $p=0.5$ basis and test functions, a quadratic-curved model used with $p=1.5$ functions, and a cubic-curved model used with $p=2.5$ functions. Ideal asymptotic rates of $O\left(h^{3}\right), O\left(h^{5}\right)$, and $O\left(h^{7}\right)$ are shown for comparison.

Figures 9 and 10 show EFIE results for the SCS error for the same torus, plotted versus the average edge length. The $p=0.5$ and $p=2.5$ results converge somewhat slower than the ideal rates of $O\left(h^{3}\right)$ and $O\left(h^{7}\right)$, while the $p=1.5$ data appear to closely follow the $O\left(h^{5}\right)$ rate. Figure 10 shows that the convergence rate for the $p=2.5$ results is slower than $O\left(h^{5}\right)$ if quadratic-patch models are used, while it is between $O\left(h^{6}\right)$ and $O\left(h^{7}\right)$ when cubic-patch models are used.

\section{Conclusions}

Far-field convergence rates are studied using two curved targets. Numerical solutions of the EFIE and MFIE are employed with higher-order vector basis functions and target models that involve flat facets, quadratic-curved patches, or cubic-curved patches. Superconvergence in the SCS at rates close to those suggested in [5] is observed in the results, provided that the models in use have high enough curvature, and the testing integration is done with quadrature rules of sufficient accuracy.

An interesting result of these observations is that it appears necessary to employ cubic-curved-patch models in order to obtain ideal convergence rates with quadratic basis functions and the MFIE. This is at odds with the classical literature on numerical methods where it is supposed that quadratic models should be adequate with quadratic

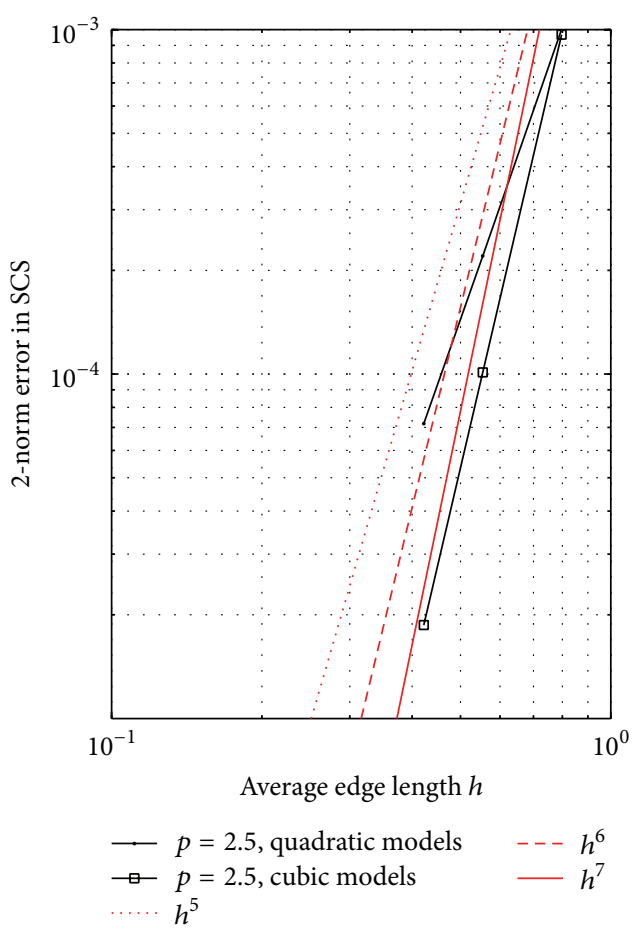

FIgURE 10: Error in the SCS versus the average edge lengths in the models, for the same torus used in Figures 7-9. EFIE results are reported for quadratic-curved and cubic-curved models used with $p=2.5$ functions. Asymptotic rates of $O\left(h^{5}\right), O\left(h^{6}\right)$, and $O\left(h^{7}\right)$ are shown for comparison.

expansions. It also appears necessary to employ cubic models with the $p=2.5$ mixed-order basis functions (which are only complete to degree 2 ) used with the EFIE to approach ideal SCS convergence rates. A similar conclusion applies to the lower-order bases; namely, the linear $p=1.0$ functions (used with the MFIE) and $p=1.5$ functions (used with the EFIE) require quadratic-patch models to yield near-ideal convergence rates.

\section{Competing Interests}

The author declares that he has no competing interests.

\section{References}

[1] R. F. Harrington, Time-Harmonic Electromagnetic Fields, McGraw-Hill, New York, NY, USA, 1961.

[2] S. Wandzura, "Optimality of Galerkin method for scattering computations," Microwave and Optical Technology Letters, vol. 4, no. 5, pp. 199-200, 1991.

[3] J. R. Mautz, "Variational aspects of the reaction in the method of moments," IEEE Transactions on Antennas and Propagation, vol. 42, no. 12, pp. 1631-1638, 1994.

[4] A. F. Peterson, D. R. Wilton, and R. E. Jorgenson, "Variational nature of galerkin and non-galerkin moment method solutions," IEEE Transactions on Antennas and Propagation, vol. 44, no. 4, pp. 500-503, 1996. 
[5] K. F. Warnick, Numerical Analysis for Electromagnetic Integral Equations, Artech House, Boston, Mass, USA, 2008.

[6] S. M. Rao, D. R. Wilton, and A. W. Glisson, "Electromagnetic scattering by surfaces of arbitrary shape," IEEE Transactions on Antennas and Propagation, vol. 30, no. 3, pp. 409-418, 1982.

[7] A. F. Peterson, "Observed baseline convergence rates and superconvergence in the scattering cross section obtained from numerical solutions of the MFIE," IEEE Transactions on Antennas and Propagation, vol. 56, no. 11, pp. 3510-3515, 2008.

[8] M. M. Bibby, A. F. Peterson, and C. M. Coldwell, "Optimum cell size for high order singular basis functions at geometric corners," Applied Computational Electromagnetics Society Journal, vol. 24, no. 4, pp. 368-374, 2009.

[9] J. C. Nedelec, "Mixed finite elements in $\mathbb{R}^{3}$, " Numerische Mathematik, vol. 35, no. 3, pp. 315-341, 1980.

[10] R. D. Graglia and A. F. Peterson, Higher-Order Techniques in Computational Electromagnetics, SciTech Publishing, Edison, NJ, USA, 2016.

[11] A. F. Peterson, "Beyond RWG/Galerkin solutions of the EFIE: investigations into point-matched, discontinuous, and higher order discretizations," in Proceedings of the 27th Annual Review of Progress in Applied Computational Electromagnetics, pp. 117120, Williamsburg, Va, USA, March 2011.

[12] O. Ergul and L. Gurel, "Investigation of the inaccuracy of the MFIE discretized with the RWG basis functions," in Proceedings of the IEEE Antennas and Propagation Society International Symposium, vol. 3, pp. 3393-3396, Monterey, Calif, USA, June 2004.

[13] Ö. Ergül and L. Gürel, "Improving the accuracy of the magnetic field integral equation with the linear-linear basis functions," Radio Science, vol. 41, no. 4, 2006.

[14] J. C. Nedelec, "A new family of mixed finite elements in $\mathbb{R}^{3}$," Numerische Mathematik, vol. 50, no. 1, pp. 57-81, 1986.

[15] A. F. Peterson, "Observed accuracy of point-tested and galerkin implementations of the volume EFIE for dielectric targets," Applied Computational Electromagnetics Society Journal, vol. 30, no. 3, pp. 255-260, 2015.

[16] A. F. Peterson, Mapped Vector Basis Functions for Electromagnetic Integral Equations, Morgan \& Claypool Synthesis Lectures, Morgan \& Claypool, San Rafael, Calif, USA, 2006.

[17] M. M. Bibby, C. M. Coldwell, and A. F. Peterson, "A high order numerical investigation of electromagnetic scattering from a torus and a circular loop," IEEE Transactions on Antennas and Propagation, vol. 61, no. 7, pp. 3656-3661, 2013. 


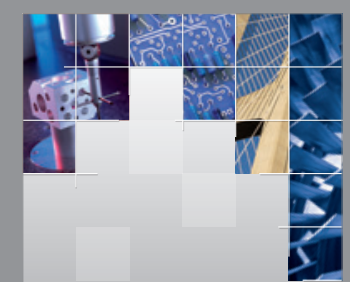

\section{Enfincering}
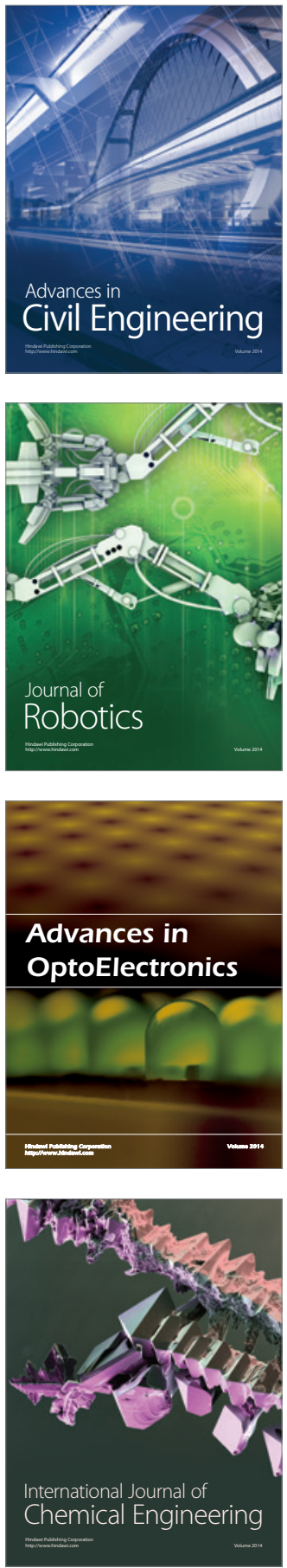

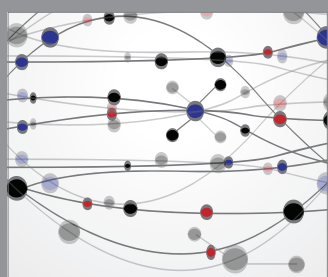

The Scientific World Journal

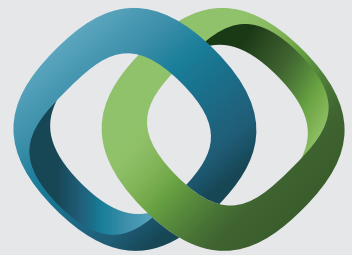

\section{Hindawi}

Submit your manuscripts at

http://www.hindawi.com
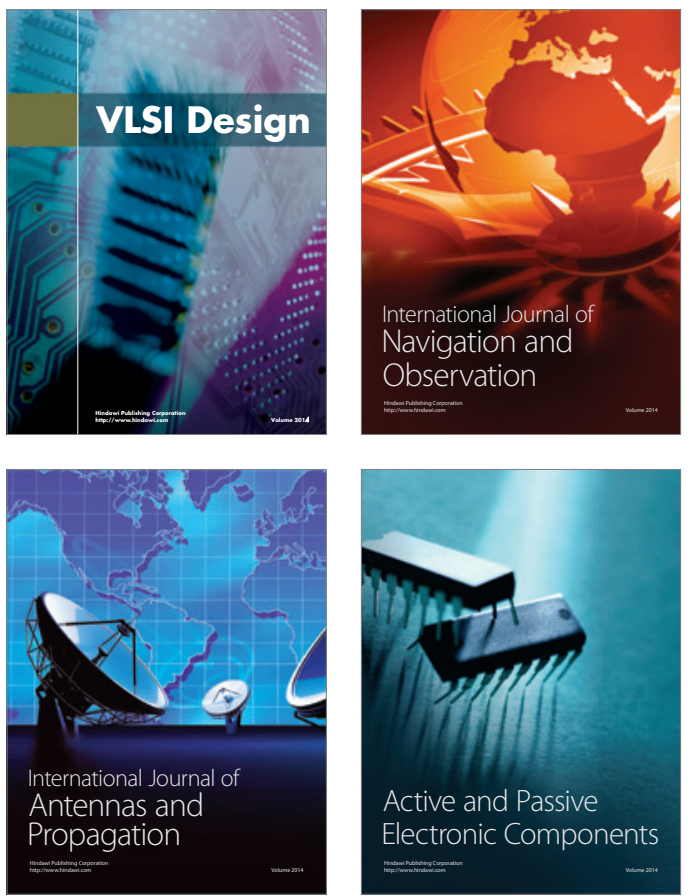
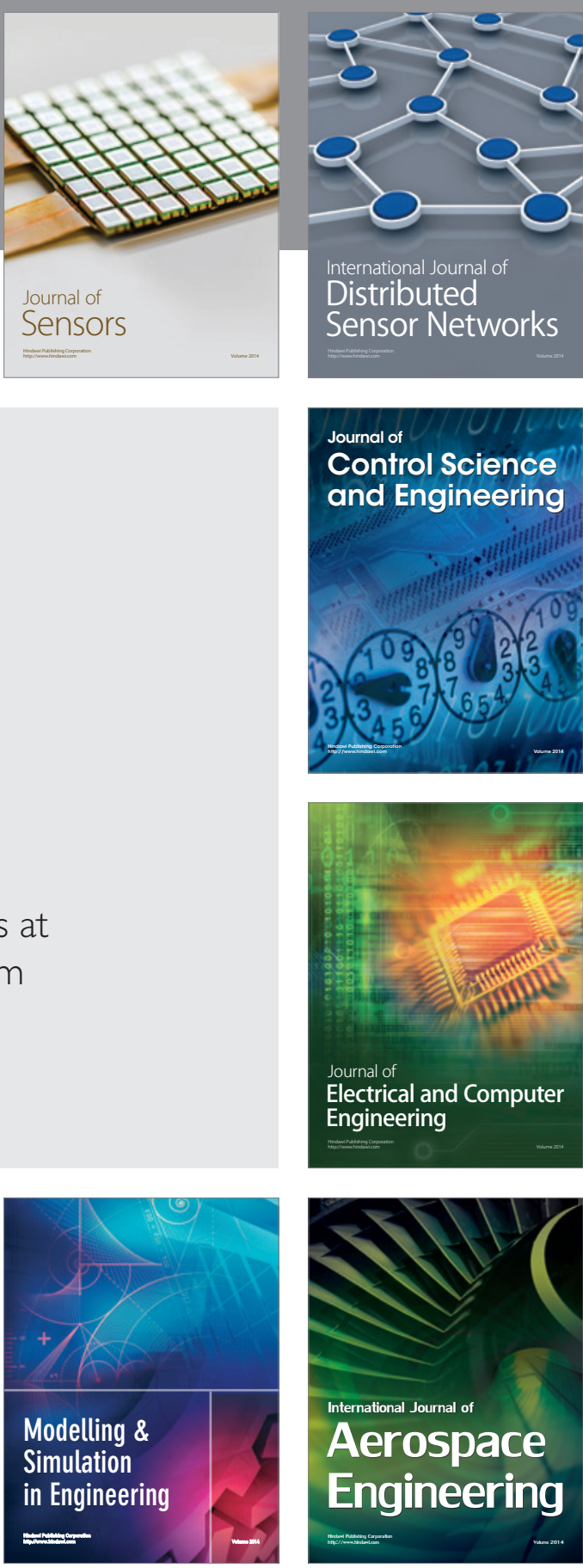

International Journal of

Distributed

Sensor Networks

Journal of

Control Science

and Engineering
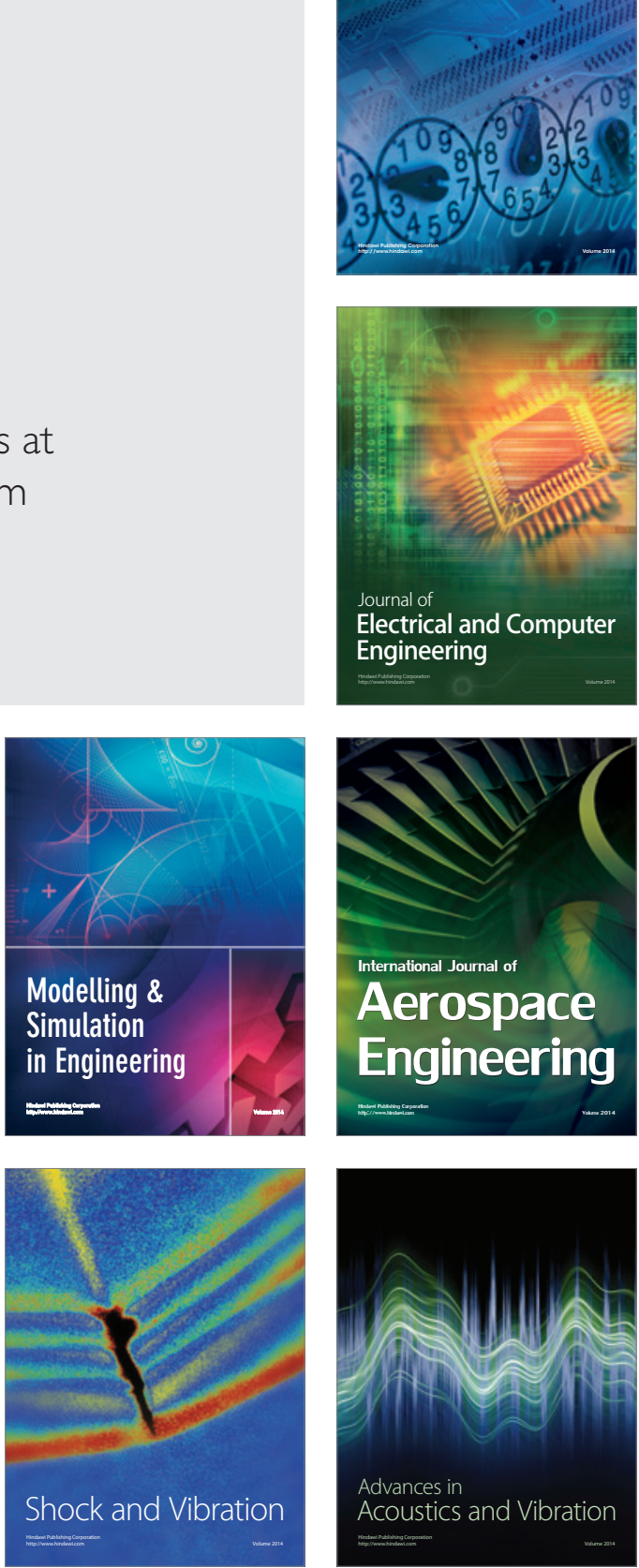